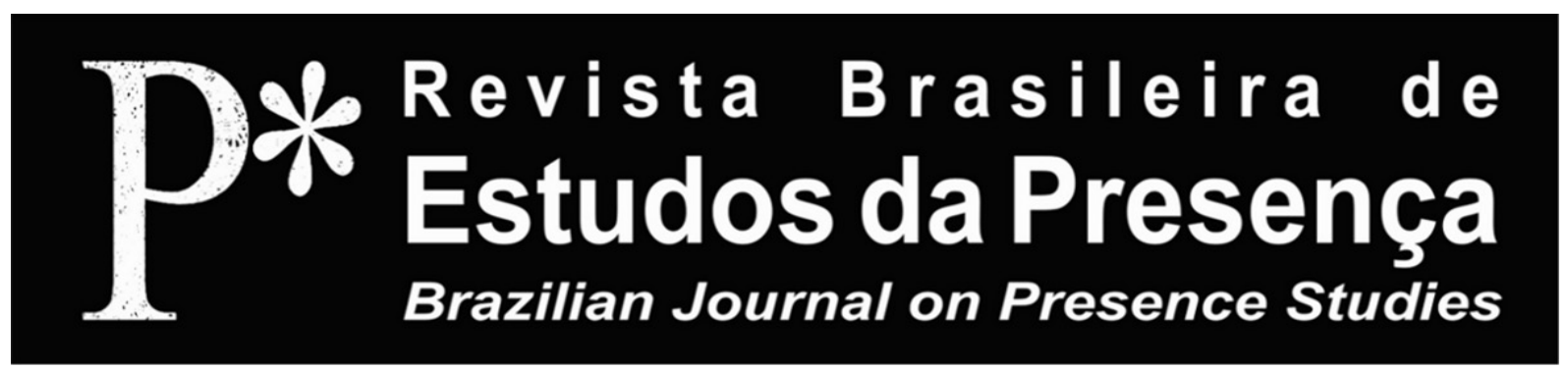

E-ISSN 2237-2660

\title{
Le Parcours Étudiant du Festival Transamériques (FTA): une expérience de médiation culturelle marquante
}

\author{
Lucie Villeneuve \\ Université du Québec à Montréal - Montréal, Canada
}

RÉSUMÉ - Le Parcours Étudiant du Festival Transamériques (FTA): une expérience de médiation culturelle marquante - Le but de cet article est de présenter les retombées éducatives du Parcours étudiant du FTA, consacré à la création contemporaine en théâtre et en danse au Québec. Une première enquête sur le terrain a été menée en 2010 auprès de 50 adolescents, suivie d'une autre, en 2014, afin de valider les résultats. La recherche nous révèle que l'évènement s'avère un rite de passage pour les jeunes; il est source de découverte de soi tout autant qu'ouverture à l'altérité, et ce, tant au niveau de l'esthétique que sur les plans culturel et socio-politique.

Mots-clés: Médiation Culturelle. Adolescents. Création Contemporaine. Rite de Passage. Ouverture à soi et à l'Autre.

ABSTRACT - Student Outreach at Festival Transamériques (FTA): a cultural mediation experience that stimulates personal transformation - The purpose of this article is to present the educational spinoffs of the Student Outreach program of the Festival Transamériques (FTA), a festival devoted to the creation of contemporary theatre and dance performance in Québec. A first field investigation was conducted in 2010 with fifty adolescents, followed by another in 2014 to validate its results. The research showed that the event acts as a kind of rite of passage for young people, serving as a source of selfdiscovery as much as well as generating openness to others at an aesthetic level and on a cultural and socio-political plan.

Keywords: Cultural Mediation. Adolescents. Contemporary Creative Arts. Rite of Passage. Openness to Self and Others.

RESUMO - O Percurso Estudantil do Festival Transamériques (FTA): uma experiência de mediaçáo cultural marcante - Este artigo visa apresentar os impactos educativos do percurso estudantil do Festival Transamériques (FTA), dedicado à criaçáo contemporânea em teatro e dança no Quebec. Uma primeira pesquisa de campo foi feita em 2010 junto a cinquenta adolescentes, seguida de outra, em 2014, com o objetivo de validar os resultados. A pesquisa revela que o evento representa um ritual de passagem para os jovens; ele é fonte tanto de descoberta de si quanto de abertura ao outro em nível estético e nos planos cultural e sociopolítico.

Palavras-chave: Mediaçáo Cultural. Adolescentes. Criaçáo Contemporânea. Ritual de Passagem. Abertura a si e ao Outro. 


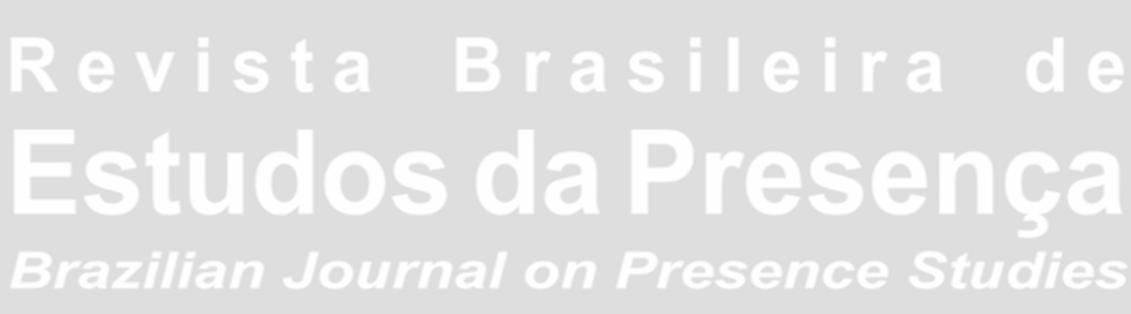

E-ISSN $2237-2660$

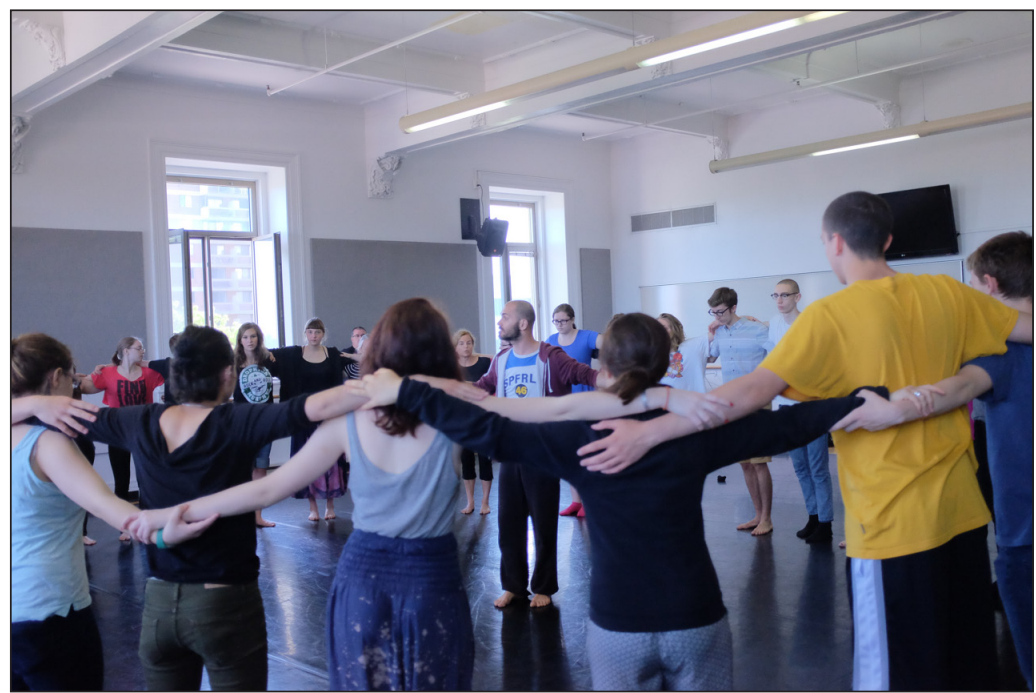

Image 1 - Parcours étudiant du FTA 2014. Atelier de danse. Photo: Sandrick Marhurin.

Dans le champ de l'éducation artistique pour les jeunes, le Festival Transamériques (FTA) a mis sur pied un programme d'activités extra-scolaires afin d'initier les adolescents à des esthétiques contemporaines. Le Parcours étudiant du FTA, implanté en 2001, présente une formule inédite de partenariat culture-école qui s'inscrit dans le paradigme de la «médiation culturelle», une expression qui est apparue en France au cours des années 1990, et qui occupe de plus en plus une place de choix au Québec (Lafortune, 2012, p. 9).

Bien que le terme médiation soit souvent accolé au domaine de la culture, il est utilisé depuis plusieurs années, tant dans le champ de l'intervention sociale et pédagogique que dans le domaine artistique, afin de traduire le caractère complexe des dispositifs de la communication. En théâtre, plus spécifiquement, la substitution du terme de communication à celle de médiation permet d'aller au-delà du processus artistique: «[...] elle est transmission et production du sens», témoigne de «[...] phénomènes idéologiques auxquels la transmission donne lieu» (Caune, 1996, p. 35) car "[...] si le théâtre peut être la scène du monde, c'est moins parce qu'il représente le monde d'une manière artistique que parce qu'il met en jeu l'intersubjectivité et la donne à voir dans l'expérience humaine» (Caune, 1996, p. 48).

À cet espace de médiation entre soi et l'autre qui loge au cœur de la pratique artistique, s'ajoutent des modes divers de médiation culturelle qui visent un travail de proximité entre le spectateur, l'œuvre et l'artiste. Ils ont pour but de démocratiser la culture et d'instaurer ainsi de nouvelles formes de partage du savoir: 
Par delà la fonction de relations publiques destinée à l'élargissement des publics, la médiation culturelle incarne un souci d'améliorer la qualité de la relation avec les œuvres, ce qui suppose un accompagnement allant d'une préparation préalable à un retour sur les expériences, et s'étend ultimement à l'engagement dans la communauté (Lafortune, 2012, p. 212).

Lorsque le FTA met sur pied son volet Parcours Etudiant en 2001 , il entend poursuivre auprès des adolescents la voie dans laquelle il s'était engagé dès son entrée sur la scène montréalaise, en 1985. Le FTA est considéré alors comme un évènement international de théâtre contemporain dont on salue le "caractère audacieux» de sa programmation «[...] et son ouverture à des théâtralités hybrides et à des formes transgressives» (David, 2009, p. 126). Ces choix vont grandement influencer la formation des publics car l'on entend aller plus loin que la simple diffusion ou la production des spectacles:

S'il est un aspect fondamental de l'importance du FTA depuis bientôt vingt-cinq ans, c'est sa politique d'intégration aux productions programmées d'un vaste ensemble d'activités: rencontres avec les créateurs, lectures publiques, projection de documentaires [...]. Ces échanges n'ont rien d'accessoire, car il ne s'agit pas d'aligner des spectacles, fussent-ils exceptionnels. Il faut encore leur donner les moyens d'avoir une résonnance réflexive dans la sphère publique. Les spectateurs du FTA sont dès lors conviés à une fête des sens, dans toute la portée sémantique du terme (David, 2009, p. 127).

En 2007, au moment où le FTA jumelle le théâtre et la danse et choisit l'appellation Festival Transamériques ${ }^{1}$ la programmation du Parcours Etudiant se diversifie: le séjour culturel vise dorénavant à initier les élèves du second cycle du secondaire à la création contemporaine, tant dans le domaine du théâtre que de la danse. Or, la création contemporaine, très hétérogène, ne se laisse pas si aisément définir: «Le terme «contemporain` a, depuis plus d'une dizaine d'années, évincé ceux de moderne et de postmoderne comme appendice lexical privilégié pour désigner la pratique artistique actuelle» (Lesage, 2012, p. 37).

Par son invitation à plonger dans des expériences esthétiques fortes, le Parcours étudiant du FTA incite les adolescents à faire un "pas de côté» (Sarrazac, 2002, p. 23) dans l'univers de la scène contemporaine où le spectacle nécessite un mode de réception plus intuitif. D’après Lehmann, d'ailleurs, le poète de l'image qu'est Wilson, représenterait l'exemple le plus marquant de la thèse selon laquelle une véritable communication artistique «[...] ne s'opère pas par la compréhension, mais par des impulsions pour la créativité personnelle du récepteur: 
des impulsions dont la communicabilité est ancrée dans les prédispositions universelles de l'inconscient» (Lehmann, 2002, p. 102). Ainsi, les scènes créées par Wilson «[...] ne veulent être ni interprétées ni comprises de manière rationnelle. Elles sont destinées à déclencher des associations, à stimuler une productivité propre dans le «champ magnétique entre la scène et le spectateur» (Lehmann, 2002, p. 103). Cette posture rejoint un grand nombre d'artistes contemporains du théâtre et de la danse qui, depuis la fin du $\mathrm{XX}^{\mathrm{e}}$ siècle, proposent des créations variées qui sont le résultat de diverses combinatoires:

À mesure que les métissages artistiques se multiplient, des auteurs travaillent à s'imaginer de nouvelles places, soit en occupant simultanément plusieurs fonctions de la création, soit en faisant du texte un partenaire original de l'image, de la musique, de la danse. Les anciennes pratiques n'ont évidemment pas disparu, mais elles tendent à devenir un processus de travail parmi bien d'autres (Ryngaert, 2011, p. 69).

Dans ce contexte de démocratisation de la culture, la mise sur pied du Parcours Étudiant constituait un véritable défi pour les organisateurs du FTA.

\section{Méthodologie de la Recherche}

Dans le but de contribuer à l'avancement des connaissances en médiation culturelle auprès d'un public d'adolescents, notre recherche a voulu examiner la structure du programme du Parcours Étudiant du FTA et jauger son impact sur les jeunes festivaliers. Plus spécifiquement, elle a visé à analyser les retombées éducatives du Parcours Etudiant du FTA pour les éditions 2010 et 2014 auprès des élèves participants. Afin de valider les résultats, nous souhaitions également comprendre son évolution à l'intérieur de ce cadre temporel, étant donné l'intervalle de quatre ans qui sépare les deux étapes de la recherche.

En lien avec la théorie de Schaeffer (2008) selon laquelle tous les types d'apprentissage peuvent être exploités en art, nous avons choisi de faire une recherche de type qualitatif, avec questions ouvertes, afin d'influencer le moins possible les réponses des participants (Karsenti; Savoie-Zajc, 2011).

[...] dans la mesure où il existe une diversité de processus cognitifs et de types d'acquisition cognitive, on ne peut pas se contenter d'une définition générale de la cognition: il existe des connaissances qui sont à un niveau pré-attentionnel, alors que d'autres sont le résultat de processus 
attentionnels, une connaissance procédurale n'est pas la même qu'une connaissance déclarative, une connaissance propositionnelle n'est pas la même chose qu'un savoir-faire, un know-how. Or tous ces processus et types d'acquisition cognitive peuvent être exploités, entre autres, par les œuvres d'art (Schaeffer, 2008, p. 74).

La collecte des données s'est faite auprès d'une cinquantaine d'élèves (provenant des écoles Armand-Corbeil, de Terrebonne, SaintLouis, de Montréal, Chavigny, de Trois-Rivières), de leurs enseignants accompagnateurs et des organisateurs des activités pédagogiques du FTA pour l'édition 2010. Nous avons également rencontré une cinquantaine d'élèves du Collège de Champigny, de Québec, de l'école Chavigny, de Trois-Rivières, et de l'école Saint-Louis, de Montréal, pour l'édition 2014.

Afin de maximiser l'efficacité de notre collecte de données, nous avons choisi plusieurs approches auprès des jeunes: ce procédé de triangulation a permis de valider nos réponses. Ainsi, les jeunes festivaliers ont été observés de façon globale tout au long de leur Parcours, en tant que spectateurs et en tant que participants aux divers ateliers, ainsi que lors des discussions animées par les médiateurs du FTA et les artistes invités. Nous avons également privilégié les entretiens informels avec les jeunes, en plus de l'analyse du journal de bord dans lequel étaient consignés les commentaires personnels et les appréciations des spectacles auxquels ils avaient assisté ${ }^{2}$. Les données que nous avons collectées auprès des jeunes festivaliers ont été fort éclairantes pour saisir la portée de la médiation culturelle mise sur pied par le FTA.

\section{État des Lieux: le Parcours Etudiant du FTA}

Le Parcours Étudiant du FTA connaît une belle réception depuis ses débuts. Une vingtaine d'écoles du Québec, dont certaines situées en région éloignée, ont participé à cet évènement qui constitue un métissage de la culture et de l'éducation. Plus de mille élèves ont pu visiter des œuvres majeures du répertoire contemporain, tout en étant initiés à la démarche de leurs créateurs grâce aux rencontres avec les artistes, ateliers, conférences et discussions qui jalonnent l'évènement, échelonné sur quatre jours. Outre les cinq spectacles en danse et en théâtre et les ateliers pratiques et tables rondes qui sont prévus à l'agenda, les élèves sont conviés à des visites au Musée d'Art Contemporain, à la découverte du plus vieux théâtre de la ville de Montréal ${ }^{3}$ encore 
en fonction, ainsi qu'à des visionnements de films liés à la programmation, activités qui convient les jeunes à une diversité de pratiques artistiques. Le Parcours entend participer au développement de toutes les dimensions de la personne et-inscrire l'éducation artistique dans le vaste champ de la dimension socio-politique puisqu'il vise à:

[...] contribuer à faire de chaque spectateur un citoyen averti, sensible aux témoignages de créateurs de tous les continents; un citoyen mieux armé pour apprécier des œuvres contemporaines, des démarches d'artistes qui osent, dénoncent, témoignent, magnifient, transgressent, bouleversent, et observent sans concession nos sociétés (Festival Transamériques, 2014, n.p.).

Le type de médiation proposé par le FTA s'appuie sur une collaboration étroite avec le milieu scolaire établie dès la phase préparatoire du projet.

\section{La Phase Préparatoire au Festival}

Plusieurs mois avant la tenue de l'évènement, des réunions préparatoires sont prévues avec les enseignants accompagnateurs afin de partager les informations relatives au contenu des spectacles, aux activités pédagogiques, et à la logistique du parcours étudiant. Cette étroite concertation avec les responsables du milieu scolaire, qui débute dès le choix de la programmation, vise à faire en sorte que l'expérience artistique des jeunes soit accompagnée d'un encadrement pédagogique de qualité. Une grille de lecture des spectacles de danse et de théâtre est alors remise par les responsables de l'encadrement pédagogique du FTA 4 .

En parallèle, les enseignants prévoient dans leur planification annuelle des moments pour préparer leurs élèves au festival. Ils développent alors la compétence liée à l'appréciation ${ }^{5}$ d'ouvres dramatiques et chorégraphiques issues de la création contemporaine. La grille de lecture des spectacles de danse et de théâtre, remise plus tôt par le FTA, fait alors partie intégrante du cahier artistique (ou journal de bord) des élèves, exigé par les enseignants, afin qu'ils puissent garder les traces de leurs appréciations, de leurs commentaires personnels et de leurs analyses critiques. Pour ce qui a trait aux retombées éducatives, cette étape est cruciale car la grille constitue un support didactique utile à l'apprentissage: elle présente les éléments de contenu et cerne les enjeux liés à la réception de l'œuvre.

Selon les enseignants accompagnateurs, la préparation en classe constitue une étape déterminante dans le Parcours Étudiant du FTA. 
Elle vise à susciter l'intérêt de l'élève, à stimuler sa curiosité et son ouverture, et à l'amener à mobiliser les connaissances qu'il a déjà intégrées à propos du théâtre ou de la danse. Cette préparation le place en état de réceptivité face à l'œuvre et décuple son expérience de spectateur.

Lors des entrevues, les enseignants accompagnateurs spécifient que cet état d'esprit serait différent de celui qu'ils observent lors des sorties régulières au théâtre tout au long de l'année scolaire. Le Parcours Étudiant du FTA vient créer chez les élèves un engouement, il est attendu dans la hâte et la curiosité de découvrir des artistes qu'il serait impossible de connaître ou de côtoyer autrement. Certains enseignants accompagnateurs précisent que les élèves s'attendent à être happés par l'énergie des artistes, à être bousculés par leurs propositions artistiques fortes.

On constate donc aisément que lors de l'élaboration du contenu du Parcours, la complicité entre les responsables pédagogiques et leur vis-à-vis du milieu scolaire fait en sorte que le terrain pour les apprentissages est bien préparé. Soulignons également une entente financière entre les parties qui vient faciliter la participation à l'évènement: les élèves bénéficient d'un tarif privilégié pour les spectacles ainsi que pour les nuits d'hébergement aux résidences étudiantes de l'Université du Québec à Montréal (UQAM).

\section{Les Rencontres et les Ateliers Préparatoires en Lien avec les Spectacles (Éditions 2010 et 2014)}

Tout au long du festival, les ateliers et les discussions qui précèdent ou suivent les spectacles au programme constituent également des moments essentiels pour renforcer les apprentissages en art dramatique et en danse. Des ateliers de démarrage sont animés par les médiateurs GilPhilippe Pelletier ${ }^{6}$ (art dramatique) et Fabienne Cabano ${ }^{7}$ (danse) qui accompagnent les groupes d'élèves tout au long du parcours. Ces activités visent à introduire certaines notions liées aux pratiques actuelles des arts de la scène et à démystifier l'art contemporain. Elles sont en général très appréciées des élèves et des enseignants accompagnateurs.

Dès le début du Parcours, les médiateurs du FTA animent une activité d'accueil dont le but est de casser la glace et de faciliter le contact entre les élèves des différentes écoles. Cette activité de démarrage plonge les festivaliers dans de courtes séquences de création, stimule l'engagement et la prise de risque, et encourage les élèves à prendre la parole lors des échanges et des discussions qui vont suivre. 
Fabienne Cabano introduit dès le départ des notions qui recoupent des éléments de théorie dont nous avons traité plus haut. Elle invite les jeunes festivaliers à regarder le spectacle de danse contemporaine en restant toujours attentifs à leurs sensations. Elle précise que le spectateur est actif, et que l'acte de regarder commande «[...] une ouverture d'esprit, une souplesse, un non-jugement et une sensibilité-radar»(Villeneuve, 2014, n.p.) Lors de l'édition 2014, elle conseille aux jeunes de lire au préalable le programme de la pièce Built to Last de Meg Stuart, qu'ils verront le soir même, et d'observer, lors de la représentation, les réactions des interprètes à la musique, ainsi que leur vulnérabilité. Elle leur propose également d'analyser les qualités physiques et théâtrales des danseurs, qui sont différentes pour chacun, les types de gestuelle, et l'originalité de la scénographie. Enfin, élément fondamental: elle traite de la nécessité d'appliquer le non-jugement, en salle, comme en atelier; ne pas juger et ne pas se juger. Cette préparation constitue une belle amorce aux autres ateliers du Parcours, les jeunes festivaliers étant invités au départ à avoir une attitude d'ouverture aux différentes propositions artistiques.

\section{Les Ateliers avec les Artistes en Lien avec la Programmation}

Au cours du festival les jeunes suivent des ateliers avec des artistes de renommée internationale. Les artistes invités proposent aux élèves des pistes d'exploration; ils les initient à leurs processus de création et les plongent ainsi au cour même de leur univers. Mieux que quiconque, le créateur donne accès à sa vision du monde, à son énergie et à sa sensibilité artistique. Ces rencontres laissent une vive impression chez les jeunes, peu importe la réception qu'ils se font de l'œuvre, de l'artiste ou de sa démarche artistique.

Lors de l'édition 2010, quoique certains élèves aient été décontenancés par le rythme et le type de composition de More More More... Future, un spectacle de danse africaine avec des accents punks, ils ont été impressionnés par l'engagement politique et artistique des chorégraphes Salia Sanou et Faustin Linyekula, qui ont présenté leur approche dans le cadre d'une rencontre préparatoire au quartier général du festival. Plus que le théâtre intime des deux artistes, c'est le théâtre du monde qui était ici convoqué. D’après Fausin Linyekula, le fait de faire de la danse contemporaine dans un pays comme le Congo, où règne le désenchantement, et d'oser être optimiste est en soi un acte révolutionnaire. Pour les adolescents, cette prise de position qui fait de la création un geste de résistance teinté d'optimisme a été une grande 


\section{( \\ Estudos da Presenca \\ Brazilian Journal on Presence Studies}

source d'étonnement. Les élèves, très réceptifs au discours des deux chorégraphes, ont été touchés par leur capacité de résilience.

\section{L'Incorporation des Apprentissages: l'élève-spectateur devient créateur}

En plus des périodes de discussion, les festivaliers ont également eu droit à des ateliers pratiques animés par les chorégraphes québécois Sylvain Émard ${ }^{8}$ et Frédérick Gravel ${ }^{9}$, des artistes qui sont en principe plus proches de leur réalité québécoise mais qui leur ont fait également visiter d'autres territoires. Les jeunes élèves en art dramatique avaient à se familiariser à un langage gestuel différent de celui du théâtre ainsi qu'à un autre type d'exploration du corps, de l'espace et de l'objet. Initiative heureuse de la part de Sylvain Émard, il a fait répéter la dernière séquence du Très Grand Continental, que les jeunes ont eu le plaisir de danser lors d'un rappel en compagnie de la centaine de danseurs amateurs qui se produisaient sur la Place Émilie-Gamelin au centre-ville de Montréal. Ce fut pour les adolescents un moment festif qui leur a permis d'occuper la place publique et la ville de manière inhabituelle:

Alors que le sentiment d'appartenance à une communauté est peut-être moins puissant dans l'anonymat des grandes villes, ces spectacles le rendent de nouveau sensible au citoyen qui, saisi dans une fiction urbaine en plein air, prend ici conscience de son inscription au sein d'une entité collective qui le dépasse. Un spectacle comme Le Très Grand Continental invite ainsi leurs participants à faire l'expérience d'un décloisonnement éphémère où les différences de catégories s'entrelacent dans un tissu social renouvelé. Sur des rythmes de danses aussi variées que le $\mathrm{R} \& \mathrm{~B}$, la valse, la techno, la disco ou encore le country, tout ce que la société comporte de catégories (sexuelles, géographiques, générationnelles) se trouve ici résorbé dans un joyeux métissage (Siaud, 2010, p. 102)

Par ailleurs, dans l'atelier animé par le chorégraphe Frédérick Gravel, les élèves ont dû prendre des risques et vaincre une certaine pudeur. Après un échauffement exigeant axé sur le contact physique et la conscience de l'espace, ils ont poursuivi avec la création de séquences avec objets (chaises), et de séries de déplacements inspirés du spectacle de danse Tout se Pète la Gueule, Chérie qu'ils avaient vu la veille. La conception d'ateliers liés de près à la programmation permet au spectateur d'intérioriser l'esthétique du spectacle et de littéralement incorporer les apprentissages car l'élève-spectateur devient créateur. Il s'agit ici d'une expérience esthétique qui est totale. 
Les entrevues que nous avons menées en 2010 avec les élèves à propos des ateliers de danse nous ont permis de saisir à quel point ce médium a constitué une découverte importante pour ceux qui sont issus d'options ou de concentrations en art dramatique. Garçons et filles ont mentionné avoir été étonnés par leur capacité d'abandon, leur lâcher-prise, résultant selon eux du climat de confiance instauré par le jeune chorégraphe Gravel qui a su éveiller leur admiration tout en étant accessible. Ces expériences fortes ont suscité un réel intérêt pour la danse; plusieurs jeunes ont exprimé le désir d'aller voir des spectacles de danse contemporaine.

Pour l'édition 2014, qui comptait pour la première fois un groupe d'élèves dont la concentration artistique est la danse, le sens critique est plus aiguisé. Les élèves ont salué l'originalité de plusieurs productions et ont déploré, tout comme certaines critiques officielles du festival, les faiblesses de certaines représentations: «En revanche, il fallait souvent, pour y arriver, subir des longueurs, des errances, des provocations qui, bien qu'elles aiguisent le sens critique, finissent par user la patience» (Doyon, 2014, n.p.).

Centré sur une initiation aux états de corps, à la manière de Meg Stuart ${ }^{10}$ et aux diverses sensations que procure la musique, l'atelier de danse animé par Katya Montaignac ${ }^{11}$, en lien avec le spectacle Built to Last, qui avait été vu trois jours plus tôt, a été bien accueilli, certains l'ayant même préféré au spectacle car «[...] l'on comprend mieux après l'avoir expérimenté» et «[...] c'était plus agréable à faire qu'à regarder» (Villeneuve, 2014, n.p.). Quant à l'atelier animé par l'un des danseurs de l'Association Fragile de la compagnie de Christian Rizzo ${ }^{12}$, il a mené les élèves à un travail d'intériorisation et de lâcher-prise qui s'est avéré plus difficile pour certains mais qui a tout de même été apprécié par la majorité des festivaliers. Certains types d'exercices s'avèrent en effet très exigeants pour les élèves; les jeunes réalisent alors à quel point il est difficile d'aller au-delà des habitudes du quotidien et de laisser une partie de leur corps guider leur mouvement, plutôt que la tête.

\section{Les Ateliers de Théâtre et de Performance}

Côté théâtre, en 2010, dans un atelier préparatoire à la trilogie de Wajdi Mouawad ${ }^{13}$ le médiateur GilPhilippe Pelletier a puisé ses ressources du côté de la para-littérature et de la pédagogie de l'indirect ${ }^{14}$ : les élèves devaient présenter en simultanéité un texte nonthéâtral (lettre écrite par Mouawad à ses acteurs), et une séquence 
gestuelle avec didascalies imposées (rituel). Comme le but était de créer un décalage entre le texte lu et le rituel joué, de nouveaux effets de sens étaient créés.

Ce type d'atelier, modulé autrement en 2014 dans le cadre d'un atelier préparatoire au spectacle Les Particules Élémentaires, mis en scène par Julien Gosselin ${ }^{15}$, a permis aux jeunes festivaliers de comprendre les effets de l'aléatoire, procédé très souvent utilisé dans la création contemporaine. Les quelques extraits qui ont été joués par les élèves lors de l'atelier préparatoire ont pris une toute autre dimension dans le contexte réel de la représentation théâtrale, car le fait de reconnaître ces partitions et de les entendre cette fois-ci avec l'ensemble du texte de la pièce, venait accentuer leur portée sémantique.

Dans le même ordre d'idées, lors du Parcours de 2010, le dramaturge québécois Étienne Lepage ${ }^{\mathbf{1 6}}$ a fait travailler les élèves à partir d'une liste de mots-contraintes qu' ils devaient intégrer à la création de récits ou de dialogues lors d'un atelier d'écriture. Contrairement à ce qu'ils pensaient ou à ce qu'ils avaient anticipé, les jeunes ont été surpris de constater que les contraintes d'écriture stimulent l'imaginaire de l'écrivain. Face au défi posé par la contrainte, l'écriture emprunte des voies auxquelles ils n'auraient sans doute pas songé autrement.

L'édition 2014 du FTA a proposé de nouvelles tendances en esthétiques contemporaines. Plusieurs représentations mettaient en avant le travail de performeurs, dans une hybridation de textes narratifs et de gestes du quotidien chorégraphiés. En lien avec cette esthétique, les élèves ont été conviés par les fondatrices du Système Kangourou, à une initiation à la performance et à un travail sur la présence. Dans l'un des micro-groupes, les objets contenus dans les sacs à dos des élèves étaient répandus sur le sol; au cours de leur déambulation au sein de chacune des équipes de création, les spectateurs qui s’arrêtaient devant cette installation étaient invités à classer les objets en catégories préétablies, un clin d'œil à la pièce Germinal, dans laquelle les performeurs avaient créé un mode de classement absurde, la catégorie des choses qui font poc poc. Dans cette hybridation de l'intime et du public, les jeunes dépassent ainsi les frontières du théâtre et de la danse pour aller vers des territoires inconnus. Soulignons finalement que pour les deux éditions, le même engouement s'est produit lors de l'atelier donné au Musée d'Art Contemporain. S'il a été pour plusieurs festivaliers une révélation, il a su éveiller la curiosité de la majorité d'entre eux.

On constate donc aisément que les deux éditions du Parcours Étudiant qui ont fait l'objet de cette recherche se caractérisent tant 
par l'audace de la programmation que par la finesse de l'approche pédagogique, étant donné le lien étroit entre les ateliers et le contenu des spectacles qui sont programmés. En plus de démystifier l'art contemporain, la structure du Parcours donne des pistes de lecture à l'appréciation d'œuvres parfois qualifiées d'inclassables et qui, autrement, seraient difficilement accessibles. L'encadrement pédagogique élaboré par le FTA reste donc fidèle aux objectifs de la médiation théâtrale du FTA, tels qu'énoncés par Amélie Côté lors d'une entrevue qu'elle accordait à Ney Wendell en 2011, alors qu'elle était responsable de la médiation:

La médiation théâtrale, comme toute médiation culturelle, repose principalement sur la transcription et la transmission des démarches artistiques adaptées aux différents publics, en fonction de leur âge, de leur bagage culturel, de leur culture et leurs connaissances. Il s'agit de rapprocher les univers, celui du créateur à celui du récepteur, en présentant les éléments des démarches artistiques de façon adaptée et vivante. On espère que ces nouvelles connaissances pousseront le récepteur à se questionner davantage sur la question traitée, à pousser ces propres réflexions sur le contexte de présentation des œuvres, historique et politique, notamment (Côté apud Wendell, 2012, p. 196).

\section{La Réception des Spectacles}

Dans le cadre d'un festival à la programmation aussi audacieuse, certains spectacles interpellent plus que d'autres les adolescents. En 2010, le coup de cœur des festivaliers a été Le Sang des Promesses de Wajdi Mouawad, une trilogie qui conviait les festivaliers à un marathon de douze heures. Viennent ensuite le spectacle de danse Tout se Pète la Gueule, Chérie, annoncé comme étant provocateur et dont les scènes de nudité n'ont pourtant pas scandalisé les adolescents, ainsi que L'Effet de Serge, du Français Philippe Quesne ${ }^{17}$, un spectacle iconoclaste créé autour du vide puisqu'il ne se passe presque rien. Ces trois spectacles, prisés par les élèves, composaient pourtant avec des esthétiques fort différentes. Trait général, cependant, les jeunes aiment l'audace: ils ont été impressionnés par la démesure de Mouawad, la non-censure de Gravel et l'esthétique insolite de Quesne.

Pour ce qui est du tout dernier Parcours, le réjouissant Germinal, signé par le tandem franco-belge Antoine Defoort et Halory Goerg $^{18}$, a créé un réel engouement tant chez les adolescents que chez les critiques, par son humour et son invention. Dans leur recherche philosophico-théâtrale de l'origine du langage ainsi que de la genèse 
du langage scénique, les performeurs ont littéralement défoncé la scène avec des pioches et questionné, par ce geste spectaculaire, les codes du théâtre. D’autre part, la moitié des festivaliers ont aimé le spectacle très attendu Les Particules Élémentaires, inspiré du roman de Houellebecq et mis en scène par Julien Gosselin, dont la conférence avait par ailleurs été accueillie chaleureusement. Ils ont été touchés par la thématique de la pièce (la misère sexuelle), la scénographie épurée, et la non-censure (les nombreuses scènes de nudité). Quant au spectacle de danse contemporaine D'après une Histoire Vraie, de Christian Rizzo, inspiré par le souvenir d'une séquence de danse folklorique turque, une bonne partie des festivaliers ont apprécié la fraternité qui se dégageait de ce groupe de danseurs masculins, la fluidité de la gestuelle, ainsi que la puissance de la musique rock des deux batteurs. Les jeunes vibrent aux propositions artistiques fortes, audacieuses et rythmées.

\section{Le Spectacle de la Ville}

La ville est "[...] un espace de sociabilité», «[...] un espace de la vie politique [...]mais aussi un espace de culture, de savoir et de signification" (Lamizet, 2002, p. 17). Pour compléter l'inventaire des retombées éducatives du Parcours Étudiants du FTA, ajoutons à la liste, la découverte de Montréal. Les jeunes festivaliers ont eu droit à une visite guidée du Monument-National, animée par Jean-Marc Larrue, professeur et historien du théâtre, qui leur a fait découvrir, outre l'historique de l'édifice, l'histoire de Montréal sur plusieurs décennies.

La ville a été également découverte de l'intérieur par les jeunes du fait qu'ils logeaient aux résidences situées dans le Quartier des spectacles pendant quatre jours et qu'ils ont pu parcourir la ville à pieds lorsqu'ils se rendaient d'un lieu à l'autre pour leurs activités. Ces nombreuses marches dans Montréal ont permis aux adolescents de s'approprier l'espace de la ville et de lui donner du sens (Lamizet, 2002, p. 11). Le séjour en résidences a également eu un impact important pour eux puisqu'il s'agissait en quelque sorte de leur première expérience en appartement. Pour plusieurs, ce fut une étape déterminante. C'est ce que révèle l'analyse de leur journal de bord dans lequel sont consignés leurs commentaires personnels. Cette expérience laisse chez certains un souvenir tout aussi vif que le plus beau des spectacles auxquels ils ont pu assister. 


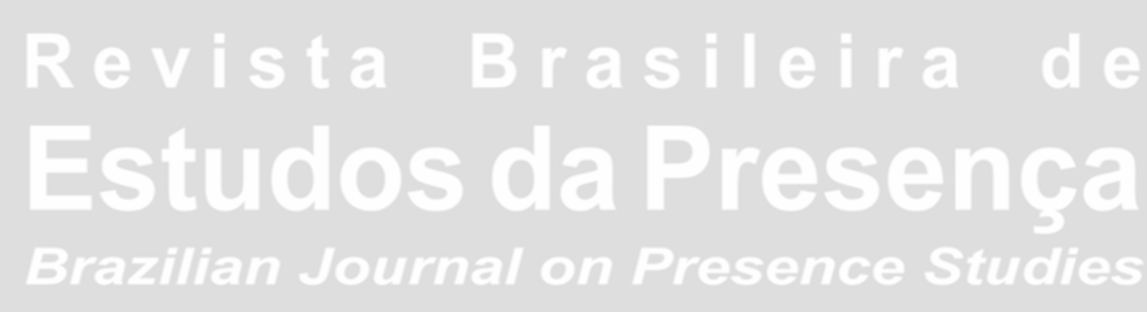

\section{Conclusion}

Quelques mois après la fin de l'édition 2010 du Parcours, les enseignants accompagnateurs nous révèlent que leurs élèves de l'option ou de la concentration art dramatique, qui sont maintenant en cinquième secondaire, voient le théâtre et la danse sous de nouveaux angles et saisissent mieux les enjeux de la création; ils estiment que le Parcours Étudiant du FTA agit comme un véritable catalyseur auprès de leurs élèves. Leur imaginaire étant nourri, ils ont encore plus le goût de créer et d'être audacieux dans leurs créations. Cela se traduit par le désir d'explorer des esthétiques jamais expérimentées auparavant et d'oser prendre des risques.

Pour ce qui est du Parcours Étudiant de l'édition de juin 2014, l'enthousiasme de ce groupe de participants y est aussi vif que chez les festivaliers de 2010, et le sens critique semble s'être aiguisé. Bien qu'il s'agisse de groupes d'élèves différents, l'évaluation qualitative du parcours est positive pour les éditions 2010 et 2014, et demeure très similaire, si l'on en juge par les apprentissages réalisés.

Les jeunes festivaliers ont fait un grand nombre d'apprentissages tout au cours de leur Parcours qu'ils ont qualifié de «marquant» dans le sens noble où l'entendait le sociologue Fernand Dumont: «[...] c'est ma situation dans le monde qui est modifiée; l'espace de ma vie ne sera plus tout à fait le même» (Dumont, 1968, p. 55-56). Dans les faits, le type de connaissances acquises va varier d'un individu à l'autre «[...] selon sa capacité ou sa volonté de s'engager plus ou moins sérieusement dans une relation cognitive avec les œuvres en question" (Schaeffer, 2008, p. 75). Cette notion fondamentale de l'engagement peut d'ailleurs se lire dans le sous-texte des écrits des festivaliers.

Sur le plan cognitif, l'ouverture d'esprit est l'énoncé qui revient le plus souvent. On mentionne également la redécouverte du théâtre et la découverte d'autres formes artistiques, telles que la danse, la performance (ou le théâtre, pour les élèves provenant de la concentration danse). Certains ajoutent que l'expérience du Parcours Étudiant $d u$ FTA leur a permis d'acquérir du vocabulaire artistique et certaines connaissances relatives à la scénographie. La notion de lâcher-prise évoquée par Fabienne Cabano lors des ateliers préparatoires à la réception des spectacles semble avoir porté ses fruits car certains notent dans leur bilan, qu' ils ont appris à «[...] apprivoiser le registre contemporain» et à "[...] s'abandonner lors de la réception d'un spectacle afin de rendre l'expérience plus riche» (Villeneuve, 2014, n.p.). Sur 
le plan artistique, les apprentissages en lien avec l'affectif sont liés au plaisir de l'évènement et à la réception des spectacles. Leur portée cognitive résulte d'un processus complexe car les émotions esthétiques, en plus de permettre "[...] d'échapper aux limites de la condition temporelle» (Jouve, 2001, p. 27), agissent de façon subtile sur le plan sémantique "[...] parce qu’elles nous rendent capables de discerner, de distinguer des aspects subtilement signifiants d'une œuvre» (Morizot, dans Darsel et Pouivet, 2008, p. 55). La programmation variée du Parcours Etudiant du FTA aura multiplié les occasions de faire vivre aux jeunes des émotions esthétiques fortes, le festival ayant été décrit à maintes reprises comme étant impressionnant.

En plus du voir, les jeunes mentionnent également avoir beaucoup apprécié les ateliers qui leur ont donné l'occasion d'apprendre par le faire. Il s'agissait ici d'une expérience esthétique totale, le genre d'expérience qui devrait, d'après Kerlan (2004), loger au centre du processus éducatif puisqu'il engage l'être tout entier.

Sur le plan social, enfin, le Parcours Étudiant du FTA a été particulièrement stimulant. Les adolescents ont appris à «[...] ne pas avoir peur de l'inconnu qui peut être magnifique» (Villeneuve, 2014, n.p.). Ils mentionnent avoir pris de la maturité. D'autres sont fiers d'être devenus moins timides, ou encore avouent avoir appris «[...] à ne pas être en retard» (Villeneuve, 2014, n.p.) et à gérer leurs déplacements dans la ville de Montréal. Ces habiletés sociales constituent des jalons importants dans l'acquisition de leur autonomie et facilite leur passage vers la vie adulte.

Ainsi, le Parcours Étudiant du FTA constitue en quelque sorte un rite de passage pour les jeunes dans lequel se multiplient les rencontres de toutes sortes: avec les œuvres et les créateurs de divers pays, avec d'autres formes artistiques, avec les élèves d'autres écoles, et, sur une base plus personnelle, avec leurs pairs qu'ils apprennent à connaître autrement, dans un contexte qui n'est pas celui de l'école ni celui de la maison. Toutes ces expériences humaines, loin d'être négligeables, contribuent à faire de leur Parcours une expérience qui les confronte à d'autres réalités et les transforme. Bien ancré dans le contexte des pratiques actuelles en théâtre et en danse, l'encadrement pédagogique pluridisciplinaire du FTA s'avère source de découverte de soi tout autant qu'ouverture à l'altérité, tant au niveau de l'esthétique que sur les plans culturel, social et politique.

Nos enquêtes sur le terrain, menées en 2010 et en 2014, nous permettent d'affirmer que le type de médiation culturelle mis sur 
pied au sein du Parcours Étudiant du FTA, agit comme un véritable catalyseur dans le cheminement artistique des jeunes festivaliers, étant donné la qualité des activités artistiques vécues en amont des spectacles qui font partie de la programmation du Parcours. Par ailleurs, qu'ils aient été conçus par des artistes du Québec ou d'ailleurs, les spectacles du FTA constituent autant d'occasions pour les jeunes de s'ouvrir à d'autres univers, de découvrir d'autres sensibilités artistiques et d'aller à la découverte de soi.

Créées dans un esprit de rupture, de transgression des codes et sous le signe de l'audace, les productions du Festival Transamériques sont en résonnance avec l'énergie des adolescents, leur curiosité et leur vivacité. Les élèves participant au Parcours Etudiant du FTA ont donc la capacité de s'y reconnaître, d'adhérer ou non aux propositions artistiques, et de construire un discours réflexif sur les enjeux artistiques et sociaux de la représentation. "L'art et la culture [étant] tout à la fois, et chacun de manière spécifique, des produits du lien social et des conditions d'existence de ce lien» (Wallach, 2006, p. 120), on comprend l'importance de continuer à créer pour les jeunes des projets de médiation culturelle signifiants et complexes qui, en empruntant le registre du sensible, contribuent à la fois au développement de la personne et à son inscription dans la société.

Si les apprentissages faits dans ce contexte de médiation, en périphérie du milieu scolaire, sont aussi signifiants pour les jeunes, cela est probablement dû au fait qu'ils s'inscrivent dans le cadre d'un évènement, à l'extérieur des territoires habituellement fréquentés. Le mot évènement voulant dire fait marquant, il s'agit d'une activité qui touche, qui marque la mémoire. La dimension extra-quotidienne de l'évènement est ici à prendre en compte, tout comme c'est le cas dans d'autres types d'évènements culturels, tels les voyages ou d'autres expériences signifiantes que peuvent vivre les adolescents. Ils constituent des étapes importantes dans leur vie, des moments de transition qui viennent modifier leur relation au monde et les ouvrir à l'altérité. À tout coup, ils en sortent transformés. 


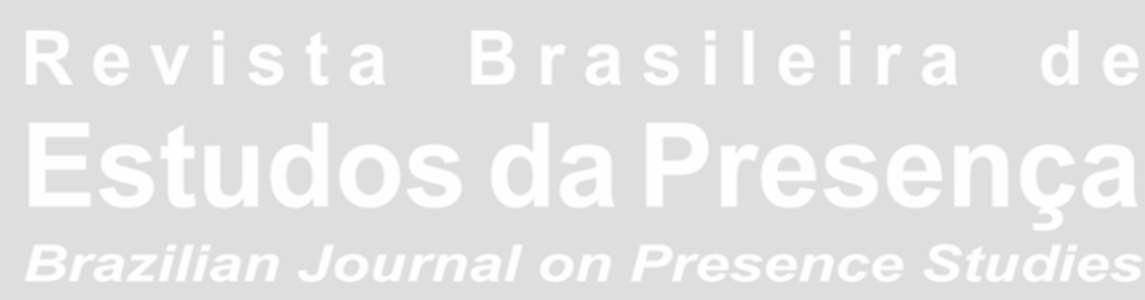

\section{Notes}

${ }^{1}$ Auparavant le festival ne concernait que le théâtre et se nommait le Festival de Théâtre des Amériques.

${ }^{2}$ Lors de l'été 2014, un questionnaire a été utilisé en remplacement du journal de bord étant donné le court délai dont nous disposions. Il était élaboré ainsi: Quels sont les premiers mots qui te viennent à l'esprit en pensant à ton Parcours du FTA? Quel évènement t'a le plus marqué? Pourquoi? Quels ont été tes spectacles préférés? Pourquoi? Peux-tu en faire la description? Que penses-tu des conférences et des ateliers qui étaient prévus à l'horaire? En bref, quels apprentissages as-tu réalisés tout au cours de ton Parcours?

${ }^{3}$ Il s'agit du Monument-National, érigé entre 1891 et 1893; il est situé sur la rue SaintLaurent, l'une des artères principales de la ville de Montréal.

${ }^{4}$ Les grilles de danse et de théâtre sont très complètes; elles présentent plusieurs pistes d'observation du spectacle ainsi que des éléments d'interprétation de l'œuvre dramatique ou chorégraphique qui aident l'élève à saisir l'ensemble des éléments de la représentation (corps, espace, scénographie, son, voix).

${ }^{5}$ La compétence apprécier est l'une des trois compétences associées au Domaine des arts du Programme de Formation de l'École Québécoise. Les deux autres compétences sont liées à la création et à l'interprétation d'œuvres dramatiques ou chorégraphiques pour les programmes d'études en art dramatique ou en danse.

${ }^{6}$ GilPhilippe Pelletier est un artiste québécois qui travaille depuis plusieurs années dans le domaine de la médiation théâtrale pour le FTA. Dramaturge et formateur, il est également chargé de cours en art dramatique à l'Université du Québec à Montréal.

${ }^{7}$ Chroniqueuse culturelle, Fabienne Cabano collabore avec divers médias montréalais, dont Voir Montréal, Voir TV, CIBL-Radio Montréal et DF Danse. Parallèlement à ses activités journalistiques, elle transmet sa passion de la danse contemporaine en agissant à titre de médiatrice culturelle. Elle accompagne des parcours étudiants au Festival Transamériques et offre des programmes de découverte de la danse contemporaine au grand public au sein du Fab Club qu'elle a fondé en 2010.

${ }^{8}$ Sylvain Émard est un danseur et chorégraphe québécois de réputation internationale, qui a créé sa compagnie, en 1987. Reconnu pour le raffinement de sa gestuelle, il surprend par la création du Grand Continental au Festival Transamériques, en 2009.

${ }^{9}$ Le jeune chorégraphe québécois Frédérick Gravel travaille dans le domaine de la danse contemporaine au Québec, aux Etats-Unis et en Europe (Gravel Works, créé au FTA 2009 et présenté en France, aux États-Unis, et en Allemagne; Tout se pète la gueule, créé au FTA 2010, et présenté en France). Il touche à la musique, au théâtre et à la performance dans des approches qui montrent la mécanique du spectaculaire.

${ }^{10}$ La chorégraphe et danseuse américaine Meg Stuart crée ses premières pièces à New York à la fin des années 1980 et connaît un premier succès international avec Disfigure Study (1991). Elle s'installe par la suite en Belgique, puis en Allemagne, où elle crée divers projets avec des plasticiens, vidéastes, musiciens, bruiteurs et divers créateurs. 


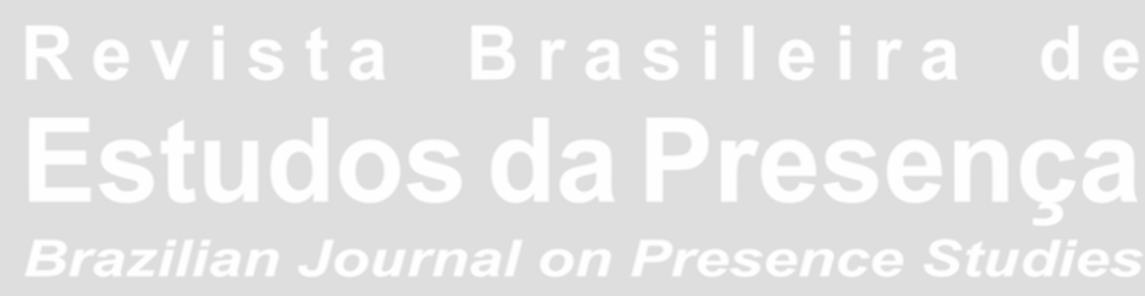

${ }^{11}$ Katya Montaignac est la fondatrice de la compagnie québécoise Objets Dansés Non Identifiés (ODNI) et directrice de nombreux projets atypiques.

${ }^{12}$ Artiste multidisciplinaire né à Cannes, Christian Rizzo œuvre dans les milieux de la mode, des arts visuels et de l'opéra, parallèlement à ses œuvres chorégraphiques.

${ }^{13}$ Dramaturge québécois d'origine libanaise, Wajdi Mouawad est auteur, metteur en scène, comédien et cinéaste. Après avoir étudié en écriture à l'École nationale de théâtre du Canada, il fonde la compagnie Ô parleur, qu'il codirige de 1990 à 1999; il dirige le théâtre de Quat'sous de Montréal de 2000 à 2004, puis le théâtre français du Centre National des Arts du Canada, à Ottawa. En 2005, il fonde la compagnie Au carré de l'hypoténuse en France où il collabore à plusieurs productions depuis. Il est récipiendaire de plusieurs prix, dont le prix du Gouverneur du Canada (2000), et obtient plusieurs reconnaissances pour son film Incendies sorti en l'an 2000. Mouawad est l'initiateur du projet Avoir 20 Ans en 2015.

${ }^{14}$ La lettre de Mouawad n'étant pas un texte de théâtre mais un texte destiné aux acteurs en lien avec la création. La pédagogie de l'indirect fait appel à des stratégies cachées ou encore liées de façon parallèle aux objectifs globaux de la situation d'apprentissage.

${ }^{15}$ Julien Gosselin est un jeune metteur en scène français dont la troupe est située à Lille. La pièce de théâtre Les Particules Elémentaires fut crée en France au Festival d'Avignon en 2013. À travers l'histoire de deux demi-frères, Michel, chercheur en biologie moléculaire, et Bruno, obsédé par le plaisir sexuel, la pièce, teintée de sarcasme et de mélancolie, raconte un pan d'histoire de la société française, des années 1960 à la fin des années 1990.

${ }^{16}$ Étienne Lepage est diplômé du programme d'écriture dramatique de l'École Nationale de Théâtre du Canada. En 2007, il coécrit, met en scène et interprète Théâtre Catastrophe avec le Nouveau Théâtre Expérimental. En 2008, sa pièce Le Mariage de Francis Camélias remporte l'Aide à la création du Centre National du Théâtre, à Paris.

${ }_{17}$ Philippe Quesne est un auteur dramatique, metteur en scène et scénographe français.

${ }^{18}$ Antoine Defoort et Halory Goerger font partie de l'Amicale de Production, une compagnie française qui crée des formes transversales, entre les arts visuels et le spectacle vivant.

\section{Références}

CAUNE, Jean. Acteur-Spectateur: une relation dans le blanc des mots. Préface: Régis Debray. Saint-Genouph: Librairie Nizet, 1996.

DARSEL, Sandrine; POUIVET, Roger. Ce que l'Art nous Apprend: les valeurs cognitives dans les arts. Rennes: Presses Universitaires de Rennes, 2008.

DAVID, Gilbert. L'Avènement du Festival de Théâtre des Amériques (1985), ou l'Inscription d'un Régime Esthétique sans Frontières. Spirale, Montréal, Spirale Magazine Culturel Inc., n. 228, p. 126-127, sept./oct. 2009. Disponible sur: <http://id.erudit.org/iderudit/1970ac>. Consulté le: 10 juin 2014.

DOYON, Frédérique; SAINT-PIERRE, Christian. Un Public Fidèle pour un Brillant Festival. Le Devoir, Montréal, juin 2014. Disponible sur: <http://www.ledevoir.com/culture/ actualites-culturelles/410446>. Consulté le: 30 juin 2014. 
DUMONT, Fernand. Le Lieu de l'Homme: la culture comme distance et mémoire. Montréal: HMH, 1968. (Collection Constances.)

FESTIVAL TRANSAMÉRIQUES. Accompagnement des Publics. Montréal, 2014. Disponible sur: <http://www.fta.qc.ca/fr/pages/accompagnement-du-public >. Consulté le: 05 juil. 2014.

JOUVE, Vincent. Poétique des Valeurs. Paris: Presses Universitaires de France, 2001. (Collection PUF.)

KARSENTI, Thierry; SAVOIE-ZAJC, Lorraine (Dir.). La Recherche en Éducation: étapes et approches. Montréal: Editions du Renouveau Pédagogique, 2011.

KERLAN, Alain. L'Art pour Eduquer? La Tentation Esthétique: contribution philosophique à l'étude d'un paradigme. Québec: Les Presses de l'Université Laval, 2004.

LAMIZET, Bernard. Le Sens de la Ville. Paris: L'Harmattan, 2002. (Collection Villes et Sociétés.)

LAFORTUNE, Jean-Marie (Dir.). La Médiation Culturelle: le sens des mots et l'essence des pratiques. Préface: Jean Caune. Montréal: Presses de l’Université du Québec, 2012.

LEHMANN, Hans-Thies. Le Théâtre Postdramatique. Paris: L’Arche, 2002.

LESAGE, Marie-Christine. Mémoire des Arts et Scène Contemporaine. L'Annuaire Théâtral, Montréal, Université du Québec à Montréal/Société Québécoise d'Études Théâtrales, n. 52, p. 37-53, oct. 2012.

L'HÉRAULT, Pierre. Le FTA Nouveau: un lieu de rencontres entre le théâtre et la danse. Spirale, Montréal, Spirale Magazine Culturel Inc., n. 216, p. 55-56, 2007. Disponible sur: <http://id.erudit.org/iderudit/10336ac>. Consulté le: 10 juin 2014.

MORIZOT, Jacques. Enjeu Cognitif et/ou Théorie Cognitive. Dans: DARSEL, Sandrine; POUIVET, Roger. Ce que l'Art nous Apprend: les valeurs cognitives dans les arts. Rennes: Presses Universitaires de Rennes, 2008. P. 49-66.

RYNGAERT, Jean-Pierre. Écritures Dramatiques Contemporaines. Paris: Armand Colin, 2011. (Collection Lettres SUP.)

SARRAZAC, Jean-Pierre. La Parabole ou l'Enfance du Théâtre. Paris: Circé, 2002. (Collection Penser le Théâtre.)

SCHAEFFER, Jean-Marie. Quelles Valeurs Cognitives pour quels Arts? Dans: DARSEL, Sandrine; POUIVET, Roger. Ce que l'Art nous Apprend: les valeurs cognitives dans les arts. Rennes: Presses Universitaires de Rennes, 2008. P. 67-81.

SCHAEFFER, Jean-Marie. Représentation, Imitation, Fiction: de la fonction cognitive de l'imagination. Dans: CHASSAY, Jean-François; GERVAIS, Bertrand (Dir.). Les Lieux de l'Imaginaire. Montréal: Liber, 2002. P.15-32.

SCHRYBURT, Sylvain. Marie-Hélène Falcon Cède les Rênes du FTA. Jeu: revue de théâtre, Montréal, Cahiers de Théâtre Jeu Inc., v. 150, n. 1, p. 84-87, 2014. Disponible sur: <http://id.erudit.org/iderudit/71617ac>. Consulté le: 20 juin 2014. 


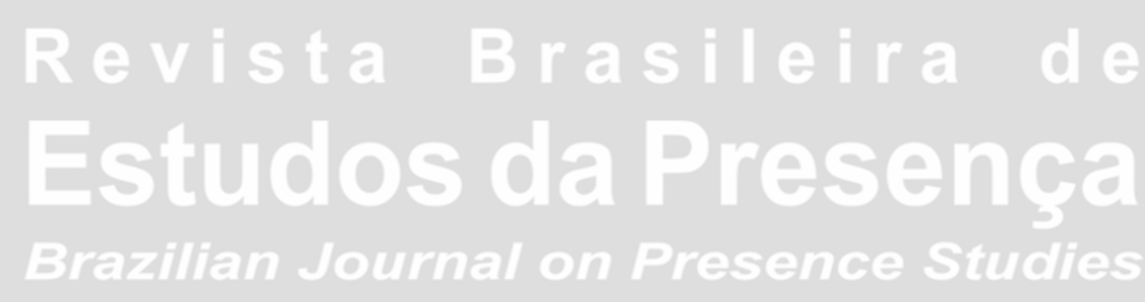

E-ISSN 2237-2660

SIAUD, Florent. Dialectique de l'Intérieur et de l'Extérieur dans Trois Evénements in situ Présentés dans l'Edition 2010 du FTA. L’Annuaire Théâtral, Montréal, Université du Québec à Montréal/Société Québécoise d'Études Théâtrales, n. 48, p. 99-112, oct. 2010.

VILLENEUVE, Lucie. Notes sur le Parcours Étudiant du FTA. Montréal, 2010.

VILLENEUVE, Lucie. Notes sur le Parcours Étudiant du FTA. Montréal, 2014a.

VILLENEUVE, Lucie. Questionnaire de la recherche auprès des adolescents. Parcours étudiant du FTA. Montréal, 2014b.

WALLACH, Jean-Claude. La Culture, pour Qui?: essai sur les limites de la démocratisation culturelle. Toulouse: Editions de l'Attribut, 2006.

WENDELL, Ney. La Médiation Théâtrale dans la Formation du Public: le projet Prends Bien Soin de Moi à Bahia et les expériences artistiques et pédagogiques dans les institutions du Québec. 2012. 230 f. Thèse (Doctorat en Etudes Théâtrales) - Programa de Pós-Graduação em Artes Cênicas, Escola de Teatro, Universidade Federal da Bahia, Salvador, 2012. Disponible sur: <https://repositorio.ufba.br/ri/handle/ri/9436>. Consulté le: 30 juin 2014.

Lucie Villeneuve, Ph.D., est professeure à l'École Supérieure de Théâtre de l'Université du Québec à Montréal et directrice de l'unité des programmes de premier cycle en art dramatique. Artiste-pédagogue à la formation multidisciplinaire, elle est diplômée en interprétation de l'École Nationale de Théâtre du Canada. Ses intérêts de recherche portent sur la médiation culturelle, les fondements philosophiques de l'enseignement du théâtre et la pédagogie des jeux dramatiques.

E-mail: villeneuve.lucie@uqam.ca

Ce texte inédit, révisé par Annelyse Gayraud, est également publié en portugais dans ce numéro.

Reçu le 4 septembre 2014 Accepté le 11 décembre 2014 